\title{
Stability Analysis of a Three-Stream Quantum-Plasma Equilibrium
}

\author{
F. Haas, \\ Laboratório Nacional de Computação Científica, LNCC \\ Av. Getúlio Vargas 333, CEP 25651-070, Petrópolis, RJ, Brazil \\ G. Manfredi, \\ Laboratoire de Physique des Milieux Ionisés, CNRS \\ Université H. Poincaré, BP 239, 54506 Vandoeuvre-les-Nancy, France \\ and J. Goedert \\ Centro de Ciências Exatas e Tecnológicas, UNISINOS \\ Av. Unisinos 950, CEP 93022-000, São Leopoldo, RS, Brazil
}

Received on 23 May, 2002

\begin{abstract}
We apply the Nyquist method to analyze the stability of small amplitude electrostatic waves in quantum plasmas described by the Wigner-Poisson system. The technique is presented in detail to assess the stability of a threestream equilibrium distribution function. The topology of the Nyquist diagrams is substantially changed with increasing quantum diffraction effects.
\end{abstract}

\section{Introduction}

The topic of quantum plasmas has received considerable attention in the recent literature [1]-[8]. The main reason for this is the increasing importance of quantum effects in the manufacturing process and performance of today's micro-electronic devices. For instance, no classical transport model can adequately incorporate quantum effects such as resonant tunnelling, which is the basis for the operation of devices like the resonant tunnelling diode. Accordingly, there has been much work dedicated to the development of quantum transport models. One of such models is provided by the Wigner-Poisson system [9, 10], which is the main concern of the present paper. We address the question of the stability of small amplitude electrostatic waves described by the Wigner-Poisson system. In the formal classical limit, the Wigner-Poisson system goes into the Vlasov-Poisson system, with linear stability properties much better understood than those of the corresponding quantum model. One natural question in this regard is: how do quantum effects modify the linear stability properties of typical classical plasma equilibria? Here we address this question in detail for the case of a three-stream plasma equilibrium.

The subject of quantum plasma stability is a subtle one. In [11] it was shown that two counter-streaming beams generate a linearly unstable mode that is not present in the corresponding classical situation. In the opposite direction, for large wave-numbers quantum effects are responsible for the suppression of all unstable modes. The ex- tra details appearing in the quantum context can be traced back to the subtle nature of quantum wave-particle interactions in a plasma. In [12], the quantum plasma instability question is addressed by means of the Nyquist method. Let $D(\omega, k)=D_{r}(\omega, k)+i D_{i}(\omega, k)=0$ be the dispersion relation for the quantum plasma, where $\omega$ and $k$ are the frequency and wave-number of linear waves, and where the dispersion function is decomposed into its real and imaginary parts. In the Nyquist method, the stability of a mode of fixed wave-number $k$ is checked by varying $\omega$ (taken as a real variable) from minus to plus infinity. Drawing the corresponding curve in the $D_{r} \times D_{i}$ plane, we obtain the socalled Nyquist diagrams. The number of unstable modes is equal to the number of times the Nyquist diagram encircles the origin. For Vlasov-Poisson plasmas, the Nyquist method shows that monotonically decreasing equilibrium functions of the energy are stable against small amplitude perturbations. Also, in this classical case the sign of the associated Penrose functional $[13,14]$ determines the linear stability of symmetric equilibria with at most two maxima. For WignerPoisson plasmas, however, there is no quantum analogue of the Penrose functional [12]. A case by case analysis must be performed, as shown in [12] for two-stream and bump-in-tail equilibria. It is the purpose of this paper to pursue in the application of the Nyquist method for simple quantum plasma homogeneous equilibrium. Here we consider a three-stream plasma and study the changes in the topology of the Nyquist diagrams as quantum effects are increased. This particular equilibrium, besides being an interesting configuration 
to test the practical applicability of the technique to systems of increased complexity, provides additional insight on how quantum effects evolve in more intricate systems.

The paper is organized as follows. In Section II we present the basic equations occurring in the Nyquist method for Wigner-Poisson homogeneous equilibria. These basic equations, derived in a recently published work [12], are included here in order to make the work self contained. In Section III we apply the Nyquist method to a three-stream equilibrium distribution function. Dimensional analysis helps to identify two essential parameters describing such classes of equilibria. Also, we identify a parameter associated with the strength of quantum effects. For this category of plasma equilibria, the real and imaginary parts of the linear dispersion relation can be exactly calculated. This helps to quickly draw the corresponding Nyquist diagrams. Holding all other parameters fixed and changing quantum effects, we observe significant changes in the topology of the Nyquist diagrams. In particular, for sufficiently large quantum effects we always observe the suppression of the linear instability. Section IV is dedicated to additional comments and conclusions.

\section{Nyquist Method and Quantum Dispersion Relations}

For $f(x, v, t)$ the Wigner quasi-distribution and $\phi$ the scalar potential, the Wigner-Poisson system $[9,10]$ reads

$$
\begin{aligned}
\frac{\partial f}{\partial t}+v \frac{\partial f}{\partial x} & =\int d v^{\prime} K\left(v^{\prime}-v, x, t\right) f\left(v^{\prime}, x, t\right), \\
\frac{\partial^{2} \phi}{\partial x^{2}} & =\frac{e}{\varepsilon_{0}}\left(\int d v f-n_{0}\right)
\end{aligned}
$$

where $K\left(v^{\prime}-v, x, t\right)$ is a functional of the scalar potential,

$$
\begin{aligned}
K\left(v^{\prime}-v, x, t\right) & =\frac{e m}{i \hbar} \int \frac{d \lambda}{2 \pi \hbar} \exp \left(\frac{i m\left(v^{\prime}-v\right) \lambda}{\hbar}\right) \times \\
& \times\left(\phi\left(x-\frac{\lambda}{2}, t\right)-\phi\left(x+\frac{\lambda}{2}, t\right)\right) \cdot
\end{aligned}
$$

This Wigner-Poisson system describes a cloud of mobile electrons immersed in a fixed ionic background. Here, $n_{0}$ is the background ionic density, $-e$ and $m$ are the electron charge and mass, $\hbar$ is the Planck constant and $\varepsilon_{0}$ is the vacuum dielectric constant. Periodic boundary conditions in space are assumed. Also, $f$ and all its velocity derivatives are assumed to go to zero for sufficiently large $|v|$. Finally, the initial Wigner function is considered to be everywhere positive. Nevertheless, the time evolution determined by Eq. (1) does not preserve the positiveness of the Wigner function, which may assume negative values. Henceforth, $f$ cannot be interpreted as a strict probability distribution function, despite its usefulness as mathematical tool to compute macroscopic quantities such as electric charge and current densities.

Let us consider a homogeneous equilibrium and small perturbations about this equilibrium. This initial value problem can be handled by Fourier transforming in space and Laplace transforming in time. This procedure yields, for the frequency $\omega$ and wave number $k$, the following quantum dispersion relation [15],

$$
D(k, \omega)=D_{r}(k, \omega)+i D_{i}(k, \omega)=0
$$

where the real and imaginary parts of the dispersion function $D(k, \omega)$ are given by

$$
\begin{aligned}
D_{r}(k, \omega) & =1-\frac{\omega_{p}^{2}}{n_{0} k^{2}} \int_{P} \frac{d v F(v)}{(v-\omega / k)^{2}-\hbar^{2} k^{2} / 4 m^{2}} \\
D_{i}(k, \omega) & =-\frac{\pi e^{2}}{\hbar \varepsilon_{0} k^{3}}\left(F\left(\frac{\omega}{k}+\frac{\hbar k}{2 m}\right)-F\left(\frac{\omega}{k}-\frac{\hbar k}{2 m}\right)\right) .
\end{aligned}
$$

In Eq. (5), $P$ stands for the principal value symbol, $F(v)$ denotes the (spatially homogeneous) equilibrium Wigner function, and, $\omega_{p}=\left(n_{0} e^{2} / m \varepsilon_{0}\right)^{1 / 2}$ is the usual plasma frequency. Notice that, no matter what the value of $\hbar,|\omega| \rightarrow \infty$ implies $D_{r} \rightarrow 1$ and $D_{i} \rightarrow 0$, as in the corresponding classical case.

Some extra analytical conclusions may occasionally be obtained. In particular, the topology of the Nyquist diagram is determined by the sign of $D_{r}$ at the points at which $D_{i}$ is zero. According to (6), these points, denoted by $v_{0}$, are the roots for $F\left(v_{0}+\hbar k / 2 m\right)=F\left(v_{0}-\hbar k / 2 m\right)$. To proceed with this strategy we must solve this equation exactly.
For the three-stream plasmas treated here, such exact solutions are not easily available and direct use of (5-6) is a more convenient approach.

Equations (5) and (6) are the basic equations for Nyquist's method for one-dimensional quantum plasmas, in which only electrostatic fields are considered. In the following we will be interested in a three-stream plasma and use (5-6) to draw the Nyquist diagrams for different values of the characteristic parameters of the equilibrium configuration and the electrostatic wave. 


\section{Three-Stream Equilibrium}

The equilibrium we shall study is specified by

$$
F(v)=\frac{\alpha n_{0} v_{1}}{\pi\left(v^{2}+v_{1}^{2}\right)}+\frac{\sqrt{2}(1-\alpha) n_{0} v_{2} v^{2}}{\pi\left(v^{4}+v_{2}^{4}\right)},
$$

where $0 \leq \alpha \leq 1, n_{0}$ is the background ion density and $v_{1}, v_{2}$ are positive constants. This distribution function describes a central beam plus two lateral, symmetric beams. In this context, $\alpha$ is the fraction of particles in the central beam, $v_{1}$ is a measure of the temperature of the central beam and $v_{2}$ a measure of the absolute velocity of the lateral beams. The cases $\alpha=0$ or $\alpha=1$, corresponding respectively to two-stream and one-stream plasmas, have been treated elsewhere [12] and will not be considered here.

The condition $F\left(v_{0}+\hbar k / 2 m\right)=F\left(v_{0}-\hbar k / 2 m\right)$, determining the zeroes of the imaginary part of the dispersion function, yields a quartic equation for $v_{0}^{2}$, and is hardly of any interest. A more efficient approach is a direct use of (5-6), after inserting the equilibrium (7). The result is

$$
\begin{aligned}
D_{r} & =1+\frac{\alpha\left(1-\Omega^{2}+H^{2}\right)}{K^{2}\left(\left(1-\Omega^{2}+H^{2}\right)^{2}+4 \Omega^{2}\right)}+ \\
& +\frac{(1-\alpha)\left(H^{2}-\beta^{2}-\Omega^{2}\right)\left(\beta^{4}-4 \beta^{2} \Omega^{2}+\Omega^{4}+H^{4}-2 \Omega^{2} H^{2}\right)}{K^{2}\left((H-\Omega)^{4}+\beta^{4}\right)\left((H+\Omega)^{4}+\beta^{4}\right)} \\
D_{i} & =\frac{2 \Omega}{K^{2}}\left(\frac{\alpha}{\left(\Omega^{2}-H^{2}\right)^{2}+2 H^{2}+2 \Omega^{2}+1}+\right. \\
& \left.+\frac{\sqrt{2}(1-\alpha) \beta\left(\left(\Omega^{2}-H^{2}\right)^{2}-\beta^{4}\right)}{\left((H-\Omega)^{4}+\beta^{4}\right)\left((H+\Omega)^{4}+\beta^{4}\right)}\right)
\end{aligned}
$$

In equations (8-9), we use the scaled variables

$$
\Omega=\frac{\omega}{\omega_{p}}, \quad K=\frac{k v_{1}}{\omega_{p}}, \quad H=\frac{\hbar k}{2 m v_{1}}, \quad \beta=\frac{v_{2}}{v_{1}} .
$$

A close examination of (8-9), shows that, as long as we are concerned with the stability analysis, $\alpha$ and $\beta$ are the essential parameters characterizing the equilibrium distribution function.

For fixed values of the scaled wave-number $K$, scaled Planck's constant $H$ and parameters $\alpha$ and $\beta$, the equations (8-9) are the parametric equations for the corresponding Nyquist diagram. This curve is obtained by varying $\Omega \in(-\infty, \infty)$. In the following two representative choices of $\alpha, \beta$ and $K$ are made and the corresponding Nyquist diagrams ploted. We vary $H$ in order to see the influence of quantum diffraction effects in the Nyquist diagrams.

First consider the case $\alpha=0.2, \quad \beta=3$. This corresponds to three streams of approximately the same intensity as shown in Fig. 1. Nyquist diagrams are plotted in Fig. 2 for $K=0.2$ and $H$ varying from $H=0$ to $H=5$. This shows that stabilization due to quantum effects is reached around $H=0.54$. From this point on no qualitative changes are observed except for topological modifications in the Nyquist diagram. This, however, does not affect the overall stability features.

Next consider the case $\alpha=0.25, \beta=3 \sqrt{2}$. In this second case we choose a configuration composed of a cen- tral beam plus two lateral warm, but less intense beams as shown in Fig. 3. For this case the Nyquist diagrams are presented in Fig 4. for $K=0.1$ and $H$ changing from $H=0$ to $H=15.0$. For this initial configuration the quantum stabilization is attained around $H=1.59$. From this point on stability is maintained and only minor topological variations on the diagrams are observed.

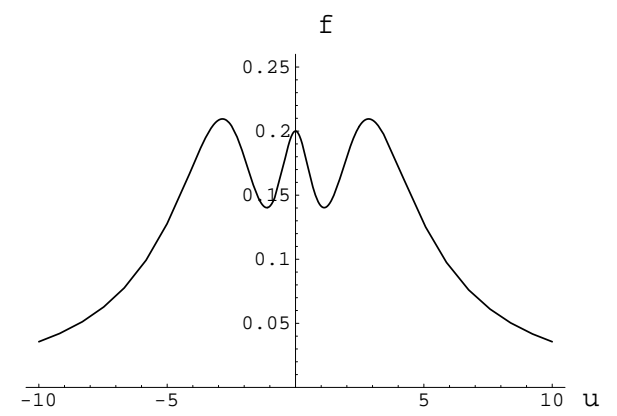

Figure 1. Three-stream equilibrium of Eq. (7) for $\alpha=0.2$, $\beta=3.0, \quad u=v / v_{1}$ and $f=F(u) /\left(n_{0} v_{1}\right)$. 

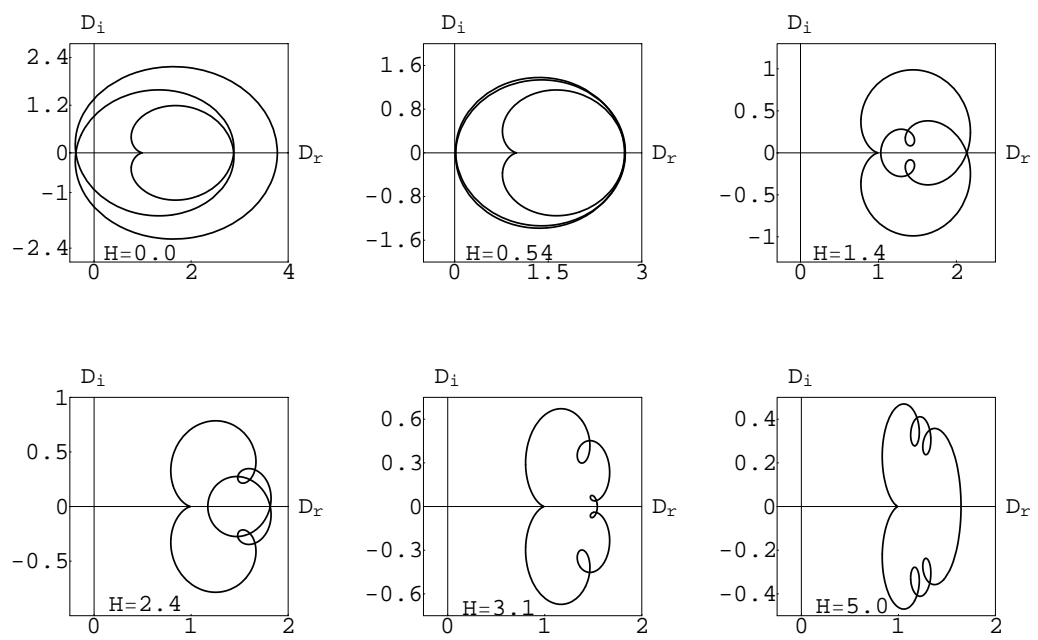

Figure 2. Nyquist diagrams for the three-stream equilibrium of Eq. (7) with $\alpha=0.2, \beta=3, K=0.2$ and $H$ as shown in the plots. The diagrams indicate that quantum effects suppress the instability for $H \geq 0.54$.

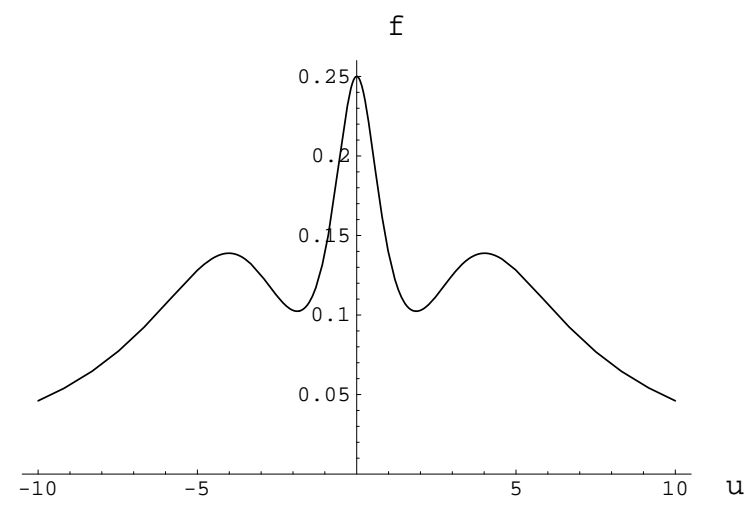

Figure 3. Three-stream equilibrium of Eq. (7) for $\alpha=0.25, \quad \beta=3 \sqrt{2}, \quad u=v / v_{1}$ and $f=F(u) /\left(n_{0} v_{1}\right)$.
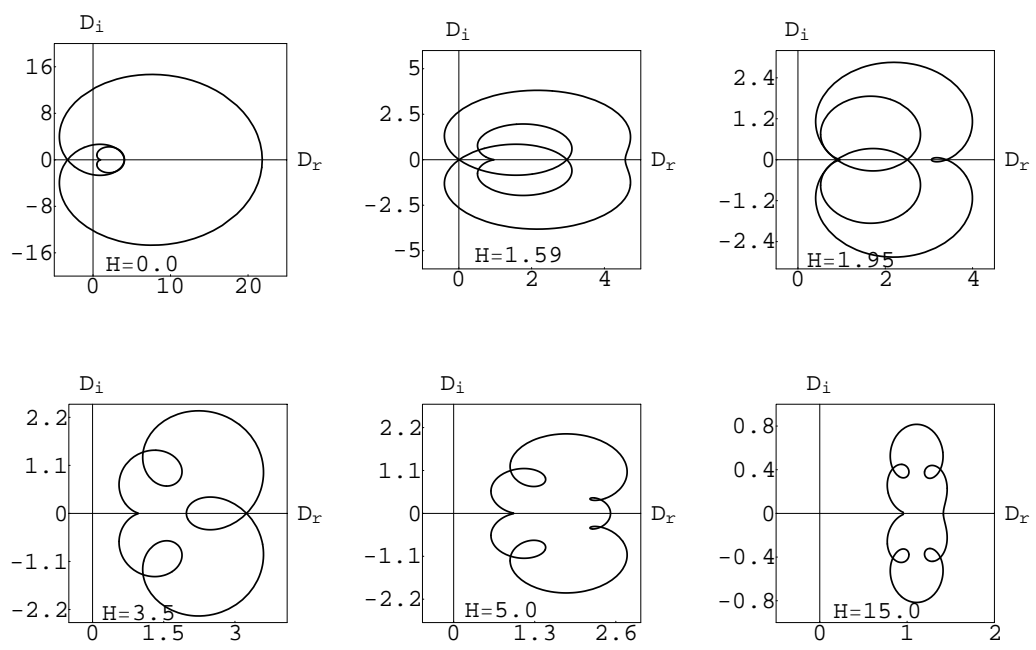

Figure 4. Nyquist diagrams for the three-stream equilibrium of Eq. (7) with $\alpha=0.25, \beta=3 \sqrt{2}, K=0.1$ and $H$ as shown in the plots. The diagrams clearly indicate that quantum effects suppress the instability for $H \geq 1.59$. 


\section{Conclusion}

In this work, the linear stability analysis of a homogeneous quantum plasma equilibria described by the Wigner-Poisson system was analyzed. Our basic tool was the Nyquist method, which provides a convenient starting point for the linear stability analysis of any dynamical system admitting a dispersion relation. We have observed how quantum effects can modify the Nyquist curves and the corresponding equilibrium stability properties of a three-stream plasma. Quantum effects are embodied in the non dimensional parameter $H=\hbar k / 2 m v_{1}$. Of course, as $\hbar$ is a fixed universal constant, changing the quantum effects is equivalent to changing $m$ (the mass of the charge carriers), $v_{1}$ (the dispersion of velocities of the central stream) or $k$ (the wave number). In this regard, looking at (5) we notice that the wavenumber enters the real part of the dispersion function not only through $\omega / k$ as for classical plasma, but also through quantum effects. Mathematically, this is responsible for the interesting changes in the Nyquist diagrams as observed in Section III, when varying $H$.

\section{Acknowledgments}

One of us (F. H.) thanks the Brazilian agency Conselho Nacional de Desenvolvimento Científico e Tecnólogico (CNPq) for financial support.

\section{References}

[1] N. C. Kluksdahl, A. M. Kriman, D. K. Ferry and C. Ringhofer, Phys. Rev. B 39, 7720 (1989).

[2] F. Cornu, Phys. Rev. E 58, 5293 (1998).

[3] B. Shokri and A. A. Rukhadze, Phys. Plasmas 6, 3450 (1999); Phys. Plasmas 6, 4467 (1999).

[4] G. Manfredi and M. R. Feix, Phys. Rev. E 53, 6460 (1996).

[5] G. Manfredi and F. Haas, Phys. Rev. B 64, 075316 (2001).

[6] S. Mola, G. Manfredi and M. R. Feix, J. Plasma Phys. 50, 145 (1993).

[7] C. L. Gardner, SIAM J. Appl. Math. 54, 409 (1994).

[8] P. A. Markowich, C. Ringhofer and C. Schmeiser, Semiconductor Equations (Springer-Verlag, New York, 1990).

[9] E. P. Wigner, Phys. Rev. 40, 749 (1932).

[10] V. I. Tatarski, Sov. Phys. Usp. 26, 311 (1983).

[11] F. Haas, G . Manfredi and M. R. Feix, Phys. Rev. E 62, 2763 (2000).

[12] F. Haas, G. Manfredi and J. Goedert, Phys. Rev. E 64, 026413 (2001).

[13] N. A. Krall and A. W. Trivelpiece, Principles of Plasma Physics (McGraw-Hill, New York, 1973).

[14] R. Penrose, Phys. Fluids 3, 258 (1960).

[15] J. E. Drummond, Plasma Physics (McGraw-Hill, New York, 1961). 